\title{
ИНИЦИАТИВА ЛЮКСЕМБУРГА SPACERESOURCES.LU И ВОЗМОЖНЫЕ ПОСЛЕДСТВИЯ ДЛЯ РЕГУЛИРОВАНИЯ МИРОВОГО РЫНКА КОСМИЧЕСКОЙ ДЕЯТЕЛЬНОСТИ
}

\begin{abstract}
Аннотация. Объект исследования - изменения в национальной космической политике государств и регулировании глобального рынка космической деятельности. Важность мониторинга этих перемен обусловливается возможностью появления неожиданных событий («черный лебедь»), способных привести к изменению сложившихся правил игры, нарушению баланса конкурентных позиций и переформатированию мировой космической экономики на новых основаниях. Предмет исследования - развитие инициативы люксембургского правительства SpaceResources.lu, связанной с созданием в рамках государственно-частного партнерства благоприятной «экосистемы» для привлечения в экономику страны компаний, специализирующихся на передовых направлениях космической деятельности. Особое внимание уделяется анализу Закона Люксембурга от 20 июля 2017 г. о разведке и использовании космических ресурсов, который является первым европейским актом, устанавливающим на национальном уровне гарантии прав собственности на ресурсы, добытые в космосе. Для решения поставленных задач были использованы общенаучные методы исследования - анализ, синтез, отслежсивание, формально-логический, сравнительно - правовой подходы. Сделан вывод о том, что появление национальных актов, нарушающих сложившиеся правила игры на рынке космической деятельности, является в определенном смысле объективно неизбежным, поскольку темпы международного правотворчества не успевают за спросом реального сектора космической экономики на новые юридические решения, способствующие его перспективному развитию. Спорные законь США и Люксембурга в области разведки и использования космических ресурсов, а особенно - деятельность люксембургского правительства в рамках инициативы SpaceResources.lu, следует рассматривать как манифестацию некой политико-правовой технологии, последовательная реализация которой с ненулевой вероятностью может привести к трансформации сложивщегося порядка в глобальной космической экономике.
\end{abstract}

Ключевые слова: Новая космическая деятельность, Космические ресурсы, Космическое право, США, Люксембург, Коммерческий космос, Договор о космосе, Соглашение о Луне, Добыча астероидов, Исследования космоса.

\begin{abstract}
The object of this research is the changes in the national space policy of countries and regulation of the global market of space exploration. The importance of monitoring these changes is justified by the possibility of emergence of unexpected events ("black swan"), capable of bringing changes to the current rules of the game, disruption in the balance of the competitive positions and reformation of the global space economy upon new terms. The subject of this research is the development of the Luxembourg initiative SpaceResources.lu associated with creation favorable "ecosystem" within the framework of state-private partnership for involvement into national economy companies that specialize in cutting-edge special exploration. Special attention is given to the analysis of Luxembourg law from July 20, 2017 on exploration and use of space resources, which represents the first European bill that establishes on the national level guarantees of rights to ownership of resources extracted from space. A conclusion is made that emergence of national legislative bills that violate the current rules of the game on the space exploration market are to a certain extent unavoidable, since the tempo of international lawmaking lags behind the demand of real sector of space economy for new legal solutions that would promote its successful development.
\end{abstract}

Keywords: New Space Activity, Space Resources, Space Law, USA, Luxembourg, Commercial Space, Outer Space Treaty, Moon Treaty, Asteroid Mining, Space research. 


\section{Исследования космоса 4(5) • 2017}

\section{Введение}

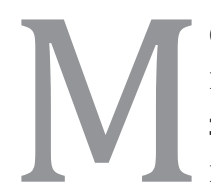

ониторинг и анализ зарубежных политико-правовых новшеств, связанных с созданием благоприятных национальных юрисдикций для космической деятельности, представляет не только теоретический, но и практический интерес в условиях реализации в Российской Федерации комплексной реформы ракетно-космической отрасли в целях «обеспечения её качественного роста» [1].

Прогресс в развитии космической деятельности считается одним из стимулов инновационного роста национальных экономик в условиях глобальной конкуренции. В свою очередь, актуальные тренды быстро меняющегося внешнего мира - интернационализация сегментов цепочек добавленной стоимости, влияние феномена «креативной деструкции», новейшие технологии и процессы (роботизация, искусственный интеллект, миниатюризация, «бережливое производство») инновативное использование традиционной продукции космического сектора с обновлением требований к ее характеристикам [2], стимулирование частной инициативы - становятся драйверами перемен как в содержании самой космической деятельности, так и в условиях ее осуществления.

Поэтому неудивительно постоянно растущее внимание к этой сфере во всем мире. Например, для того чтобы «лучше статистически идентифицировать космической сектор и исследовать его экономические измерения и инновационную роль для остальной экономики» в рамках Организации экономического сотрудничества и развития (OECD) в начале 2000-х гг. был создан Космический форум (OECD Space Forum) [3], который на регулярной основе ведет соответствующие исследования и формирует базы данных. А страны, которые исторически не имели статуса космических лидеров, ищут способы для создания и развития сектора, связанного с космической деятельностью, с тем, чтобы добиться улучшения своих инновационных показателей [см. подробнее 4].

Все эти факторы порождают поток разномасштабных изменений в глобальном политико-правовом и экономическом «вмещающем ландшафте», в котором происходит космическая деятельность. Мониторинг этих перемен крайне необходим, поскольку малозаметные «маргинальные» события могут содержать риски, ведущие к изменению сложившихся правил игры, нарушению баланса конкурентных позиций и даже к переформатированию мировой космической экономики на новых основаниях.

Характерным примером, иллюстрирующим важность комплексного отслеживания новшеств в указанной сфере, являются события (20162017), связанные с активностью правительства Люксембурга по созданию благоприятных институционально-правовых условий для привлечения в национальную юрисдикцию зарубежных компаний, специализирующихся в области передовых космических технологий.

\section{Проблема гарантий прав собственности на космические ресурсы}

Великое Герцогство Люксембург с населением менее 600 тыс. чел. стало вторым государством в мире, принявшим национальный закон о гарантиях прав собственности на ресурсы, добытые в космосе [5]. Как известно, первый шаг такого рода был сделан США - 25 ноября 2015 г. был подписан Закон о конкурентоспособности коммерческих запусков в космос (U.S. Commercial Space Launch Competitiveness Act), регулирующий деятельность частных компаний на орбите Земли и за ее пределами, а также разрешающий гражданам США свободно заниматься разработкой планет и астероидов, владеть и распоряжаться полученными ресурсами, в том числе, водой и минералами (но не живыми объектами) [6]. Принятие этого акта стало своего рода «полезной провокацией» [7], которая не только резко активизировала идущие еще с 1960-х годов теоретические споры о праве государств в одностороннем порядке регулировать вопросы собственности на «космические ископаемые» [см., например, 8-24 и др.], но и обратила внимание мирового сообщества на более общую проблему, связанную с состоянием системы международного космического права и его влиянием на перспективы развития космической деятельности. В первую очередь речь идет о поиске механизмов, позволяющих обеспечить должный баланс стабильности и творческого 


\section{Правовое регулирование космической деятельности}

развития правовых условий функционирования космической экономики, поскольку в настоящее время темпы международного правотворчества не успевают за спросом на юридические инновации, способные заложить основы регулирования будущих институтов и потенциально возможных рынков.

Продолжающиеся масштабные дискуссии по поводу Закона США о коммерческом космосе 2015 г. не привели к сближению позиций противников и сторонников этого акта [см., например, 25-36 и др.]. Однако стало ясно, что возможности его фактического функционирования, как и реальные последствия для системы международного космического права будут зависеть не столько от действий США, сколько от позиции и практических шагов других государств. Анализ событий 2016-2017 гг. показал, что, как и было предсказано [см. подробнее 37], «единая европейская оппозиция» американскому закону не сложилась, несмотря на то, что ряд европейских государств в свое время ратифицировали (Австрия, Бельгия, Нидерланды) или подписали (Румыния и Франция) Соглашение о деятельности государств на Луне и других небесных телах 1979 г. (Agreement Governing the Activities of States on the Moon and Other Celestial Bodies, коротко - «Moon Agreement») [38], которое прямо запрещает коммерческое использование космических ресурсов.

Тем самым «маргинальным» событием, которое уже повлекло за собой серию процессов, способных в перспективе трансформировать международную систему принципов и правил космической деятельности, стало решение правительства Люксембурга о запуске в феврале 2016 г. так называемой Инициативы по добыче космических ископаемых (Space Resources Mining Initiative) [39] как составного элемента национальной космической программы. Суть инициативы - создание максимально благоприятных условий (в том числе правовых) для привлечения в экономику страны космических проектов и компаний. Поэтому изначально было заявлено о намерении Люксембурга обеспечить развитие правовой базы, регулирующей права собственности на полезные ископаемые, добытые с околоземных объектов (Near Earth Objects - NEO's), в том числе астероиды [40-43]. В результате 20 июля 2017 г. был принят первый европейский Закон о разведке и использовании космических ресурсов (вступил в силу 02 августа 2017 г.) [44].

\section{Закон Люксембурга о разведке и использовании ресурсов космоса}

Закон Люксембурга формирует национальную правовую базу для «новой космической деятельности» и состоит из двух основных частей, регулирующих, соответственно, вопросы собственности на космические ресурсы и режим получения разрешения на осуществление разведки и использование таких ресурсов.

Устанавливая в ст. 1 положение о том, что космические ресурсы могут быть присвоены, законодатели, помимо прочего, исходили из аналогии с нормами, определяющими правовой режим открытого моря. В частности, речь идет о возможности свободно разведывать и добывать ресурсы открытого моря, не присваивая открытое море в целом как таковое.

Закон также установил, что для выполнения миссий по разведке и добыче космических ресурсов потенциальные операторы должны получать по письменному запросу разрешение на уровне министров правительства Люксембурга (ст. 3) и сформулировал условия получения таких разрешений. Разрешения в отношении конкретной миссии по разведке и использованию космических ресурсов в коммерческих целях предоставляются оператору на личной основе и не могут быть переданы никакому другому субъекту (пп. 2 и 3 ст. 2, а также ст. 3 и 5). Эти положения не касаются деятельности в сфере спутниковой связи, орбитальных позиций и использования полосы частот (п. 4 ст. 2). В законе также четко зафиксировано, что уполномоченный оператор обязан осуществлять свою деятельность в соответствии с международными обязательствами Люксембурга (ст. 2 п. 3). Разрешение может быть отозвано, если оператор нарушит условия, на которых оно было выдано (ст. 14).

Разрешение может быть выдано только зарегистрированным в юрисдикции Люксембурга юридическим лицам в форме акционерного общества (société anonyme), акционерно-коммандитного общества (société en commandite par actions), 


\section{Исследования космоса 4(5) • 2017}

общества с ограниченной ответственностью, созданного по люксембургскому законодательству (société à responsabilité limitée) или европейской компании (société européenne) (ст. 4). Однако акционеры или участники компании-оператора могут быть как люксембургские, так и иностранные физические или юридические лица.

Выдача разрешения на реализацию проекта по разведке и добыче космических ресурсов обусловливается строгими требованиями к профессиональной репутации компании-оператора и ее участников, к качеству ее управления и финансовой надежности. Закон, в частности, требует раскрытия информации о личности прямых или косвенных акционеров компанииоператора, владеющих 10\% уставного капитала или $10 \%$ голосов, либо о 20 крупнейших участниках (п. 1 ст. 8).

Запрос на получение разрешения, помимо детального описания космической миссии, должен содержать оценку ее рисков. В частности, от заявителя требуется точно указать объем и источники покрытия этих рисков - собственные финансовые средства оператора, полис страховой компании, либо гарантии кредитной организации, которые не должны быть аффилированы с оператором (п. 1 ст. 10).

Оператор, получивший разрешение, несет полную ответственность за любой ущерб, причиненный в ходе реализации космической миссии, а также во время всех подготовительных работ (ст. 16).

Административные расходы на рассмотрение заявки должны быть покрыты заявителем и могут составлять от 5000 до 500000 евро в зависимости от сложности работы (п. 1 ст. 13). Порядок взимания этого сбора будет регламентирован актом Великого Герцога Люксембурга (п. 1 ст. 13).

Получение разрешения на выполнение проекта по разведке и использованию космических ресурсов в коммерческих целях не освобождает оператора от необходимости получать другие необходимые в сфере космической деятельности разрешения и лицензии (ст. 17).

Заключительная статья (ст. 18) содержит перечень наказаний (штрафы, лишение свободы и пр.) за нарушение ключевых положений закона, либо условий разрешений, выданных компани- ям-операторам на осуществление космических миссий.

Международная реакция и оценки в связи с принятием люксембургского Закона от 20 июля 2017 г. о разведке и использовании ресурсов космоса оказались ожидаемо противоречивы. Одни эксперты настаивают, что это акт нарушает базовые принципы международного космического права [45-47 и др.], другие оценивают его как смелый шаг, поставивший одну из самых маленьких стран Европы в один ряд с наиболее инновативными государствами, делающими ставку на космос в развитии своей экономики [48-50 и др.].

Что касается Российской Федерации, то МИД России и Госкорпорация «Роскосмос» выступают против создания на национальном уровне правовых условий, разрешающих коммерческим компаниям разведку, добычу и присвоение ресурсов в космосе, в том числе без провозглашения права собственности на астрономические объекты (планеты, естественные спутники, астероиды, кометы и пр.), и намерены вынести на обсуждение $00 Н$ в начале 2018 г. соответствующий проект имплементационного соглашения к «Договору о космосе». Предполагается, что в случае его принятия на международном уровне будет установлен обязательный для всеобщего исполнения, не допускающий толкований, полный и однозначный запрет на присвоение космических ресурсов [51].

\section{Деятельность правительства Люксембурга по развитию инициативы SpaseResources.lu}

Несмотря на неоднозначные международные перспективы, Закон Люксембурга от 20 июля 2017 г. о разведке и использовании ресурсов космоса не только дает необходимую базу для дальнейшей реализации амбициозного проекта по созданию глобального хаба для «новой космической деятельности» (инициатива SpaceResources. $\mathrm{lu}$, но и становится аттрактором, притягивающим соответствующие ресурсы, активности, организации и идеи, способные дать синергетический эффект и, в конечном счете, переформатировать сложившуюся действительность.

Инициатива о создании в стране «особой зоны» для космической деятельности была объ- 


\section{Правовое регулирование космической деятельности}

явлена министерством экономики Люксембурга в феврале 2016 г. [52]. Уже в мае того же года было подписан первый трехсторонний протокол о государственно-частном партнерстве между люксембургским правительством, государственным банком Société nationale de crédit et d'investissement (SNCI) и компанией Deep Space Industries (DSI) в рамках инициативы SpaceResources.lu [53]. В рамках этого партнерства DSI, Inc (США) и DSI Europe (Люксембург) планируют создать пригодные для коммерциализации экономические и технологические ноу-хау, нацеленные на разведку и использование космических ресурсов, а правительство Люксембурга намерено участвовать в софинансировании перспективных проектов в рамках национальной комической программы LuxIMPULSE и программы поддержки исследований и разработок с использованием механизмов финансирования, предоставляемых SNCI (банк был создан в 1978 г. для стимулирования бизнесинвестиций, поддержки стартапов и исследовательских инициатив).

В течение года соответствующие соглашения подписали еще четыре частные фирмы, которые создали свои структуры в юрисдикции Люксембурга в рамках формирующегося глобального хаба: Planetary Resources (США) [54], ispace Europe (структура японской компании ispace, специализирующейся на космической робототехнике и лунной разведке) [55], Blue Horizon (совместная структура немецкой компании ОНВ Venture Capital GmbH и люксембургской LuxSpace) [56] и Kleos Space (100\% владелец - британская Magna Parva Limited) [57].

Параллельно Люксембург активно развивал соответствующие контакты с межгосударственными структурами и правительствами других стран. Так, например, весной 2017 г. шли обсуждения с правительством Японии возможности разработки многостороннего соглашения о разведке и использовании космических ресурсов, которое могло бы помочь привести положения Договора о космосе 1967 г. «в соответствии с реалиями дня» [58]. А в июне 2017 г. вышло в свет совместное заявление с Европейским космическим агентством об укреплении сотрудничества по проектам полетов к астероидам, развития соответствующих технологий, а также разведки и использования космических ресурсов [59].
Вступление в силу Закона от 20 июля 2017 г. позволило люксембургскому правительству укрепить переговорные позиции и привлечь новых крупных партнеров для реализации своей инициативы. Так, в октябре 2017 г. был подписан межправительственный меморандум о взаимопонимании с Объединенными Арабскими Эмиратами о партнерстве в космической деятельности [60], а в ноябре 2017 г. - двустороннее межправительственное соглашение с Японией о сотрудничестве в области разведки и использовании космических ресурсов [61]. Кроме того, в сентябре - ноябре 2017 г. меморандумы о космическом сотрудничестве и открытии компаний в юрисдикции Люксембурга заключили шведская GomSpace Group AB [62] и американская Spire Global [63]. При этом в ряде случаев люксембургские государственные структуры участвуют в учреждении новых компаний и софинансировании их деятельности. Так, например, люксембургский «Фонд Будущего» (Luxembourg Future Fund - LFF, создан государственным банком SNCI и Европейским инвестиционным фондом) будет соучредителем европейской штаб-квартиры Spire в рамках инициативы SpaceResources. lu, a Национальный Исследовательский Фонд (Luxembourg National Research Fund - FNR) принял решение о сотрудничестве с ispace Europe и финансировании двух исследовательских проектов в размере 700 млн. евро [64].

Деятельность правительства Люксембурга по «изменению правил игры»в области разведки и освоения космических ресурсов также сопровождается масштабной информационной кампанией. В частности, в ноябре 2017 г. была проведена первая международная конференция NewSpace Europe, привлекшая большое число энтузиастов «нового космоса».

\section{Выводы}

Система международного космического права, как и многие другие институты современности, в начале XXI века столкнулась с проблемой нахождения новой точки устойчивого равновесия между стабильностью и изменчивостью в условиях быстро меняющегося мира. На практике баланс между сохранением в неприкосновенности основополагающих международных догово- 


\section{Исследования космоса 4(5) • 2017}

ров о космосе и необходимостью реагировать на постоянно растущий объем новых вызовов достигается путем использования концептов «жесткого» и «мягкого» права (hard law and soft law) [65]. Однако опыт показывает, что темпы международного правотворчества не успевают за спросом на новые юридические решения, способные дать эффективный ответ на актуальные потребности космической деятельности, а тем более - заложить основы для регулирования потенциально возможных отношений и еще не созданных рынков (например, в сфере добычи и переработки космических ресурсов). В таких условиях появление национальных актов, нарушающих сложившиеся правила игры, является в определенном смысле объективно неизбежным. Законы США и Люксембурга в области разведки и использования космических ресурсов можно считать юридической «провокацией», требующей осуждения мировым сообществом. Однако анализ всего контекста происходящего показывает, что деятельность этих стран и, прежде всего, правительства Люксембурга представляет собой последовательную реализацию своего рода «подрывной» инновационной технологии (disruptive innovation) на глобальном космическом рынке. И какими бы «маргинальными» не казались инициативы одного из самых маленьких европейских государств, его репутация «надежной финансовой гавани», готовность предложить «целую экосистему и экспертные знания для привлечения капитала» [66], правительственная поддержка, развитая инфраструктура и правовая база, созданная в интересах операторов космической деятельности, в сумме дают ненулевой шанс появления устойчивой «точки роста»новой космической экономики с отличными от существующих правилами игры. В качестве одного из аргументов, демонстрирующих текущую эффективность люксембургской «инновационной технологии», можно отметить тот факт, что благодаря инициативе SpaceResources.lu доля доходов от «космического сектора»в экономике Люксембурга за один год уже возросла с нуля до 2\% ВВП [67].

\section{Библиография}

1. Вступительное слово Президента Российской Федерации на совещании о стратегии развития Госкорпорации «РОСКОСМОС». 2016. 15 ноября. URL: http://kremlin.ru/events/president/news/53261 (дата обращения: 01.12.2017).

2. The Space Economy and Space Innovation in 2016. Presentation at United Nations / United Arab Emirates High Level Forum: Space as a driver for socio-economic sustainable development - Dubai, 21 November 2016. URL: http://www.unoosa.org/documents/pdf/hlf/1st_hlf_Dubai/Presentations/38.pdf (дата обращения: 01.12.2017).

3. OECD Space Forum. URL: http://www.oecd.org/futures/oecdspaceforum.htm (дата обращения: 01.12.2017)

4. Попова С.М. Современные тенденции развития международного космического права // Право и государство (Казахстан). 2016. №4 (73) С. 66-71.

5. Loi du 20 juillet 2017 sur l'exploration et l'utilisation des ressources de l'espace // Journal officiel du GrandDuché de Luxembourg. 28.07.2017. URL: http://legilux.public.lu/eli/etat/leg/loi/2017/07/20/а674/jo (дата обращения: 01.12.2017).

6. The U.S. Commercial Space Launch Competitiveness Act of 2015. Public Law 114-90. Nov. 25, 2015. URL: https://www.congress.gov/bill/114th-congress/house-bill/2262/text (дата обращения: 01.12.2017).

7. Rummel John D. One Small Step for Space Resources // SpaceNews. December 9, 2015. URL: http://spacenews. com/op-ed-the-next-steps-for-space-resources/\#sthash.rD4m6gnw.dpuf (дата обращения: 01.12.2017).

8. Brooks E. National control of natural planet bodies - preliminary considerations // Journal of Air Law and Commerce. 1966. Vol. 32. P. 315-328.

9. Brooks E. Control and use of planetary resources // Proceedings of the Colloquium on the Law of Outer Space. 1968. Vol. 11. P. 339-360.

10. Reynolds G. International Space Law: into the Twenty-First Century // Vanderbilt Journal of Transnational Law. 1992. Vol. 25. P. 225-255. 


\section{Правовое регулирование космической деятельности}

11. Husby E. Sovereignty and Property Rights in Outer Space // Journal of Intellectual Property Law and Practice.1994. Vol. 3. P. 359-372.

12. Dunk, von der, F. Private Enterprise and Public Interest in the European "Spacescape". Towards Harmonized National Space Legislation for Private Space Activities in Europe. Leiden. 1998.

13. Benson J. Space resources: first come first served // Proceedings of the Forty-First Colloquium on the Law of Outer Space. 1999. P. 191-196.

14. Pop V. Appropriation in Outer Space: the Relationship Between Land Ownership and Sovereignty on the Celestial Bodies // Space Policy. 2000. Vol. 16 (4). P. 275-282.

15. Zullo K. M. The Need To Clarify The Status Of Property Rights In International Space Law // Georgetown Law Journal. 2002. Vol. 90. P. 2414-2444.

16. Dinkin S. Don't wait for property rights // Space Review. July 12. 2004. URL: http://www.thespacereview. com/article/179/1 (дата обращения: 01.12.2017).

17. Lewis J., Lewis C. A Proposed International Legal Regime for the Era of Private Commercial Utilization of Space // The George Washington International Law Review. 2005. Vol. 37. P. 745-767.

18. Dinkin S. Property Rights and Space Commercialization // Space Review. May 10. 2006. URL: http://www. thespacereview.com/article/141/1 (дата обращения: 01.12.2017).

19. Cherian J. G., Abraham J. Concept of Private Property in Space - An Analysis // Journal of International Commercial Law and Technology. 2007. Vol. 2. Issue 4. P. 211-220.

20. Bonin J.R., Tronchetti F. Constructing a regulatory regime for the exploitation of resources on the Moon and other celestial bodies: a balancing act // The Indian Journal of International Economic Law. 2010. Vol. 3. P. 1-27.

21. Meyer Z. Private Commercialization of Space in an International Regime: A Proposal for a Space District // Northwestern Journal of International Law \& Business, 2010. Vol. 30. Issue 1. P. 241-261.

22. Юзбашян М. Международное космическое право: Лунная «недвижимость» и шахты на астероидах // Российский космос. 2006. №3. С. 32-35.

23. Конюхова А. Новые тенденции в международном космическом праве // Право и управление. XXI век. 2010. №2. С. 82-87.

24. Вылегжанин А.Н., Юзбашян М.Р. Развитие правового регулирования экономической деятельности в космосе // Право международной безопасности: современное видение и сопутствующие проблемы межгосударственного сотрудничества. Межвузовский сборник научных трудов №3 (7). Екатеринбург, 2011. C. $91-102$.

25. Oduntan G. Who owns space? US asteroid-mining act is dangerous and potentially illegal // The Conversation. November 25, 2015. URL: https://theconversation.com/who-owns-space-us-asteroid-mining-act-is-dangerousand-potentially-illegal-51073 (дата обращения: 01.12.2017).

26. Koerth-Baker M. Who Makes the Rules for Outer Space? // NovaNext. November 30, 2015. URL: http://www. pbs.org/wgbh/nova/next/space/space-law/ (дата обращения: 01.12.2017).

27. Masson-Zwaan T., Richards B. International Perspectives on Space Resource Rights // SpaceNews. December 8. 2015. URL: http://spacenews.com/op-ed-international-perspectives-on-space-resource-rights/\#sthash. qUo6j9VQ.dpuf (дата обращения: 01.12.2017).

28. Selding, de, P. B. New U.S. Space Mining Law's Treaty Compliance May Depend on Implementation // SpaceNews. December 9, 2015. URL: http://spacenews.com/u-s-commercial-space-acts-treaty-compliance-may-dependon-implementation/\#sthash.r5TiS1gK.dpuf (дата обращения: 01.12.2017).

29. Position Paper on Space Resource Mining / International Institute of Space Law. December, 20. 2015. URL: http://www.iislweb.org/docs/SpaceResourceMining.pdf (дата обращения: 01.12.2017).

30. Tronchetti F. The Space Resource Exploration and Utilization Act: A move forward or a step back? // Space Policy. 2015. Vol. 34. P. 6-10.

31. Tronchetti F. Title IV - Space Resource Exploration and Utilization of the US Commercial Space Launch Competitiveness Act: A Legal and Political Assessment // Air and Space Law. 2016. Vol. 41. Issue 2. P. 143-156.

32. Lefeber R. Relaunching The Moon Agreement // Air \& Space Law. 2016. Vol. 41. No. 1. P. 41-48. 


\section{Исследования космоса 4(5) • 2017}

33. Алексеенко А.П. Разведка и добыча космических ресурсов: опыт законотворчества США // Юридические исследования. 2016. №5. C.34-41. DOI: 10.7256/2409-7136.2016.5.18968. URL: http://e-notabene.ru/lr/ article_18968.html (дата обращения: 01.12.2017).

34. Будылин С. Кому принадлежат астероиды? К вопросу о первоначальных способах приобретения собственности // Zakon.ru. 2016. 4 февраля. URL: https://zakon.ru/discussion/2016/2/4/komu_prinadlezhat_ asteroidy__k_voprosu_o_pervonachalnyx_sposobax_priobreteniya_sobstvennosti (дата обращения: 01.12.2017).

35. Jakhu R., Pelton J. N., Nyampong Y. O. M. Space Mining and Its Regulation. Springer Praxis Books, 2017. $180 \mathrm{p}$.

36. Юзбашян М.Р. Закон США об исследовании и использовании космических ресурсов 2015 г. и международное космическое право // Московский журнал международного права. 2017. №2 (106). С. 71-86.

37. Попова С.М. Закон США о коммерческом космосе 2015 г. и вопросы модернизации международного космического права // Исследования космоса. 2016. №1. C. 51-65. DOI: 10.7256/2453-8817.2016.1.20590. URL: http://e-notabene.ru/ik/article_20590.html (дата обращения: 01.12.2017).

38. The Agreement Governing the Activities of States on the Moon and Other Celestial Bodies. 1979. URL: http:// disarmament.un.org/treaties/t/moon/text (дата обращения: 01.12.2017).

39. SpaceResources.lu. URL: http://www.spaceresources.public.lu/en/index.html (дата обращения: 01.12.2017).

40. Messier D. Luxembourg Launches Space Resources Mining Initiative // Parabolic Arc. February 03. 2016. URL: http://www.parabolicarc.com/2016/02/03/luxembourg-launches-space-resources-mining-initiative/\#more57416 (дата обращения: 01.12.2017).

41. Messier D. Deep Space Industries Praises Luxembourg Space Resources Mining Initiative // Parabolic Arc. February 03. 2016. URL: http://www.parabolicarc.com/2016/02/03/deep-space-industries-praisesluxembourg-space-resources-mining-initiative/\#more-57417 (дата обращения: 01.12.2017).

42. Messier D. Luxembourg's Bold Move into Space Mining // Parabolic Arc. February 3. 2016. URL: http://www. parabolicarc.com/2016/02/03/luxembourgs-bold-move-space-mining/\# more-57418 (дата обращения: 01.12.2017).

43. Messier D. Luxembourg Commits \$227 Million to Space Mining Initiative // Parabolic Arc. June $08,2016$. URL: http://www.parabolicarc.com/2016/06/08/luxembourg-commits-227-million-space-mining-initiative/ \#more-58598 (дата обращения: 01.12.2017).

44. Le Luxembourg promulgue la première loi européenne sur l'exploitation des ressources spatiales // Le Monde. 31 Juillet 2017. URL:http://www.lemonde.fr/economie/article/2017/07/31/le-luxembourgpromulgue-la-premiere-loi-europeenne-sur-l-exploitation-des-ressources-spatiales_5167065_3234. html\#T6pwWxU40F4eG6hJ.99 (дата обращения: 01.12.2017)

45. Hobe S. Was Luxemburg macht, ist krass völkerrechtswidrig // Deutschlandfunk Kultur. 01 Aug. 2017. URL: http://www.deutschlandfunkkultur.de/gesetz-zu-schuerfrechten-im-all-was-luxemburg-macht-ist.1008. de.html?dram:article_id=392506 (дата обращения: 01.12.2017).

46. Luxembourg space exploration laws criticized // Luxembourg Times. 02 Aug. 2017. URL: https://luxtimes. lu/archives/1432-luxembourg-space-exploration-laws-criticised (дата обращения: 01.12.2017).

47. Белькова Л., Корчмарек Н. «Компрометирует принцип международного права»: «Роскосмос» — о законе Люксембурга о добыче ресурсов в космосе частниками // Russia Today. 05 октября 2017. URL: https://russian.rt.com/science/article/436642-roskosmos-iskopaemye-kosmos-lyuksemburg (дата обращения: 01.12.2017).

48. Schummer L., Calmes B. Luxembourg law on the exploration and use of space resources entered into force // ARENDT. 02 Aug. 2017. URL: http://www.arendt.com/publications/pages/luxembourg-law-exploration-usespace-resources-into-force.aspx (дата обращения: 01.12.2017).

49. Feider M., Levine T., Graas J. Luxembourg Space Resources Act: Paving the legal road to space // Allen\&Overy. 28 Sept. 2017. URL: http://www.allenovery.com/publications/en-gb/Pages/Luxembourg-Space-ResourcesAct-Paving-the-legal-road-to-space.aspx (дата обращения: 01.12.2017). 


\section{Правовое регулирование космической деятельности}

50. Thailly L., Schneider F. Luxembourg set to become Europe's commercial space exploration hub with new Space Law. 01 Aug. 2017. URL: http://www.ogier.com/news/the-luxembourg-space-law\# (дата обращения: 01.12.2017).

51. «Известия»: Россия предлагает запретить присвоение полезных ископаемых в космосе // ТАСС. 11 декабря 2017. URL: http://tass.ru/kosmos/4801166 (дата обращения: 01.12.2017).

52. Luxembourg to launch framework to support thr future use of space resources / Released by the Luxembourg Ministry of the Economy. 03 Feb. 2016. URL: http://www.spaceresources.public.lu/content/dam/ spaceresources/press-release/2016/2016_02_03PressReleaseAnnouncementSpaceResourceslu.pdf (дата обращения: 01.12.2017).

53. Le gouvernement du Luxembourg et Deep Space Industries ont signé un protocole d'accord pour l'exploration et l'utilisation de ressources spatiales. Communiqué - Publié le 05.05.2016. URL: http://www.gouvernement. lu/5963943/05-ressources-spatiales (дата обращения: 01.12.2017).

54. SpaceResources.lu: Luxembourg Government and Planetary Resources sign MoU to develop activities related to space resource utilization. 13 May 2016. URL: http://www.spaceresources.public.lu/content/ dam/spaceresources/press-release/2016/2016_05_13PressReleaseMoUPR-LuxGvt.pdf (дата обращения: 01.12.2017).

55. Luxembourg and ispace, a Tokyo-based lunar robotic exploration company, sign MoU to co-operate within the SpaceResources.lu initiative. 2 March 2017. URL: http://www.spaceresources.public.lu/content/dam/ spaceresources/press-release/2017/2017_03_02Pressrelease-MoU-iSpace-Lux-Gvt.pdf (дата обращения: 01.12.2017).

56. OHB Venture Capital and LuxSpace establishing company to enable sustainable life in Space. 4 Apr. 2017. URL: http://www.spaceresources.public.lu/en/actualites/2017/ohb-venture-capital-luxspace.html (дата обращения: 01.12.2017).

57. Luxembourg and Kleos Space sign a MoU to co-operate within the SpaceResources.Lu initiative // SpaceResources.Lu. 24 Jul. 2017. URL: http://www.spaceresources.public.lu/en/actualites/2017/News_Kleos_ Space.html (дата обращения: 01.12.2017).

58. Étienne Schneider to Plead in Favour of Multilateral Agreement on Exploration and Use of Space Resources. 21 Apr. 2017. URL: http://www.spaceresources.public.lu/content/dam/spaceresources/press-release/2017/201704-21-press-release-visit-japan.pdf (дата обращения: 01.12.2017).

59. Luxembourg and the European Space Agency enhance cooperation on asteroid missions, related technology and space resources exploration and utilization. 20 June 2017. URL: http://www.spaceresources.public.lu/ content/dam/spaceresources/press-release/2017/2017_06_21\%20Press\%20Release\%20ESA\%20LeBourget. pdf (дата обращения: 01.12.2017).

60. Luxembourg and the United Arab Emirates sign MoU on Space Resources. 10 Oct. 2017. URL: http://www. spaceresources.public.lu/en/actualites/2017/MoU-UAE.html (дата обращения: 01.12.2017).

61. Luxembourg and Japan agree to cooperate on exploration and commercial utilization of Space Resources. 29 Nov. 2017. URL: http://www.spaceresources.public.lu/en/actualites/2017/Luxembourg-and-Japan-Agreeto-Cooperate-on-Exploration-and-Commercial-Utilization-of-Space-Resources.html (дата обращения: 01.12.2017).

62. Luxembourg Government and GomSpace partner to develop new space activities in the Grand Duchy. 17 Sept. 2017. URL: http://www.spaceresources.public.lu/content/dam/spaceresources/press-release/2017/201709-27-press-release-mou-gomspace.pdf (дата обращения: 01.12.2017).

63. Luxembourg government and Spire Global signed cooperation agreement to open an European HQ in the Grand Duchy. 15 Nov. 2017. URL: http://www.spaceresources.public.lu/en/actualites/2017/LuxembourgGovernment-and-Spire-Global-signed-cooperation-agreement-to-open-a-European-HQ-in-the-Grand-Duchy. html (дата обращения: 01.12.2017).

64. SpaceResources.lu: First-time FNR investment in space exploration projects. 23 Nov. 2017. URL: http://www. spaceresources.public.lu/content/dam/spaceresources/press-release/2017/2017-11-23-fnr-space-funding. pdf (дата обращения: 01.12.2017). 


\section{Исследования космоса 4(5) • 2017}

65. Masson-Zwaan T. The relevance of hard law and soft law in the further development of space law. 2016. 8 Sept. URL: http://www.unoosa.org/pdf/SLW2016/Panel6/1._Masson-UN_soft_law_hard_law.pdf (дата обращения: 01.12.2017).

66. Schrieberg D. Luxembourg's Bet On Space Industry Shows Early Signs Of Success // The Forbes. 19 Nov. 2017. URL: https://www.forbes.com/sites/davidschrieberg1/2017/11/19/luxembourgs-bet-on-space-industryshows-early-signs-of-success/\#3e9984ес6114 (дата обращения: 01.12.2017).

67. The space industry is now $2 \%$ of Luxembourg's GDP, deputy prime minister Etienne Schneider says // CNBS. 11 Nov. 2017. URL: https://www.cnbc.com/2017/11/11/etienne-schneider-the-space-industry-is-now-2percent-of-luxembourgs-gdp.html (дата обращения: 01.12.2017).

\section{References (transliterated)}

1. Vstupitel'noe slovo Prezidenta Rossiiskoi Federatsii na soveshchanii o strategii razvitiya Goskorporatsii «ROSKOSMOS». 2016.15 noyabrya.URL: http://kremlin.ru/events/president/news/53261 (data obrashcheniya: 01.12.2017).

2. The Space Economy and Space Innovation in 2016. Presentation at United Nations / United Arab Emirates High Level Forum: Space as a driver for socio-economic sustainable development - Dubai, 21 November 2016. URL: http://www.unoosa.org/documents/pdf/hlf/1st_hlf_Dubai/Presentations/38.pdf (data obrashcheniya: 01.12.2017).

3. OECD Space Forum. URL: http://www.oecd.org/futures/oecdspaceforum.htm (data obrashcheniya: 01.12.2017)

4. Popova S.M. Sovremennye tendentsii razvitiya mezhdunarodnogo kosmicheskogo prava // Pravo i gosudarstvo (Kazakhstan). 2016. №4 (73) S. 66-71.

5. Loi du 20 juillet 2017 sur l'exploration et l'utilisation des ressources de l'espace // Journal officiel du GrandDuché de Luxembourg. 28.07.2017. URL: http://legilux.public.lu/eli/etat/leg/loi/2017/07/20/a674/jo (data obrashcheniya: 01.12.2017).

6. The U.S. Commercial Space Launch Competitiveness Act of 2015. Public Law 114-90. Nov. 25, 2015. URL: https://www.congress.gov/bill/114th-congress/house-bill/2262/text (data obrashcheniya: 01.12.2017).

7. Rummel John D. One Small Step for Space Resources // SpaceNews. December 9, 2015. URL: http://spacenews. com/op-ed-the-next-steps-for-space-resources/\#sthash.rD4m6gnw.dpuf (data obrashcheniya: 01.12.2017).

8. Brooks E. National control of natural planet bodies - preliminary considerations // Journal of Air Law and Commerce. 1966. Vol. 32. P. 315-328.

9. Brooks E. Control and use of planetary resources // Proceedings of the Colloquium on the Law of Outer Space. 1968. Vol. 11. P. 339-360.

10. Reynolds G. International Space Law: into the Twenty-First Century // Vanderbilt Journal of Transnational Law. 1992. Vol. 25. P. 225-255.

11. Husby E. Sovereignty and Property Rights in Outer Space // Journal of Intellectual Property Law and Practice.1994. Vol. 3. P. 359-372.

12. Dunk, von der, F. Private Enterprise and Public Interest in the European "Spacescape". Towards Harmonized National Space Legislation for Private Space Activities in Europe. Leiden. 1998.

13. Benson J. Space resources: first come first served // Proceedings of the Forty-First Colloquium on the Law of Outer Space. 1999. P. 191-196.

14. Pop V. Appropriation in Outer Space: the Relationship Between Land Ownership and Sovereignty on the Celestial Bodies // Space Policy. 2000. Vol. 16 (4). P. 275-282.

15. Zullo K. M. The Need To Clarify The Status Of Property Rights In International Space Law // Georgetown Law Journal. 2002. Vol. 90. P. 2414-2444.

16. Dinkin S. Don't wait for property rights // Space Review. July 12. 2004. URL: http://www.thespacereview. com/article/179/1 (data obrashcheniya: 01.12.2017).

17. Lewis J., Lewis C. A Proposed International Legal Regime for the Era of Private Commercial Utilization of Space // The George Washington International Law Review. 2005. Vol. 37. P. 745-767. 


\section{Правовое регулирование космической деятельности}

18. Dinkin S. Property Rights and Space Commercialization // Space Review. May 10. 2006. URL: http://www. thespacereview.com/article/141/1 (data obrashcheniya: 01.12.2017).

19. Cherian J. G., Abraham J. Concept of Private Property in Space - An Analysis // Journal of International Commercial Law and Technology. 2007. Vol. 2. Issue 4. P. 211-220.

20. Bonin J.R., Tronchetti F. Constructing a regulatory regime for the exploitation of resources on the Moon and other celestial bodies: a balancing act // The Indian Journal of International Economic Law. 2010. Vol. 3. P. 1-27.

21. Meyer Z. Private Commercialization of Space in an International Regime: A Proposal for a Space District // Northwestern Journal of International Law \& Business, 2010. Vol. 30. Issue 1. P. 241-261.

22. Yuzbashyan M. Mezhdunarodnoe kosmicheskoe pravo: Lunnaya «nedvizhimost'» i shakhty na asteroidakh // Rossiiskii kosmos. 2006. №3. S. 32-35.

23. Konyukhova A. Novye tendentsii v mezhdunarodnom kosmicheskom prave // Pravo i upravlenie. XXI vek. 2010. №2. S. 82-87.

24. Vylegzhanin A.N., Yuzbashyan M.R. Razvitie pravovogo regulirovaniya ekonomicheskoi deyatel'nosti v kosmose // Pravo mezhdunarodnoi bezopasnosti: sovremennoe videnie i soputstvuyushchie problemy mezhgosudarstvennogo sotrudnichestva. Mezhvuzovskii sbornik nauchnykh trudov №3 (7). Ekaterinburg, 2011. S. 91-102.

25. Oduntan G. Who owns space? US asteroid-mining act is dangerous and potentially illegal // The Conversation. November 25, 2015. URL: https://theconversation.com/who-owns-space-us-asteroid-mining-act-is-dangerous-and-potentially-illegal-51073 (data obrashcheniya: 01.12.2017).

26. Koerth-Baker M. Who Makes the Rules for Outer Space? // NovaNext. November 30, 2015. URL: http://www. pbs.org/wgbh/nova/next/space/space-law/ (data obrashcheniya: 01.12.2017).

27. Masson-Zwaan T., Richards B. International Perspectives on Space Resource Rights // SpaceNews. December 8. 2015. URL: http://spacenews.com/op-ed-international-perspectives-on-space-resource-rights/\#sthash. qUo6j9VQ.dpuf (data obrashcheniya: 01.12.2017).

28. Selding, de, P. B. New U.S. Space Mining Law's Treaty Compliance May Depend on Implementation // SpaceNews. December 9, 2015. URL: http://spacenews.com/u-s-commercial-space-acts-treaty-compliance-may-dependon-implementation/\#sthash.r5TiS1gK.dpuf (data obrashcheniya: 01.12.2017).

29. Position Paper on Space Resource Mining / International Institute of Space Law. December, 20. 2015. URL: http://www.iislweb.org/docs/SpaceResourceMining.pdf (data obrashcheniya: 01.12.2017).

30. Tronchetti F. The Space Resource Exploration and Utilization Act: A move forward or a step back? // Space Policy. 2015. Vol. 34. P. 6-10.

31. Tronchetti F. Title IV - Space Resource Exploration and Utilization of the US Commercial Space Launch Competitiveness Act: A Legal and Political Assessment // Air and Space Law. 2016. Vol. 41. Issue 2. P. 143-156.

32. Lefeber R. Relaunching The Moon Agreement // Air \& Space Law. 2016. Vol. 41. No. 1. P. 41-48.

33. Alekseenko A.P. Razvedka i dobycha kosmicheskikh resursov: opyt zakonotvorchestva SShA // Yuridicheskie issledovaniya. 2016. №5. S.34-41. DOI: 10.7256/2409-7136.2016.5.18968. URL: http://e-notabene.ru/lr/article_18968.html (data obrashcheniya: 01.12.2017).

34. Budylin S. Komu prinadlezhat asteroidy? K voprosu o pervonachal'nykh sposobakh priobreteniya sobstvennosti // Zakon.ru. 2016. 4 fevralya. URL: https://zakon.ru/discussion/2016/2/4/komu_prinadlezhat_asteroidy_k_voprosu_o_pervonachalnyx_sposobax_priobreteniya_sobstvennosti (data obrashcheniya: 01.12.2017).

35. Jakhu R., Pelton J. N., Nyampong Y. O. M. Space Mining and Its Regulation. Springer Praxis Books, 2017. $180 \mathrm{p}$.

36. Yuzbashyan M.R. Zakon SShA ob issledovanii i ispol'zovanii kosmicheskikh resursov 2015 g. i mezhdunarodnoe kosmicheskoe pravo // Moskovskii zhurnal mezhdunarodnogo prava. 2017. №2 (106). C. 71-86.

37. Popova S.M. Zakon SShA o kommercheskom kosmose 2015 g. i voprosy modernizatsii mezhdunarodnogo kosmicheskogo prava // Issledovaniya kosmosa. 2016. №1. S. 51-65. DOI: 10.7256/2453-8817.2016.1.20590. URL: http://e-notabene.ru/ik/article_20590.html (data obrashcheniya: 01.12.2017). 


\section{Исследования космоса 4(5) • 2017}

38. The Agreement Governing the Activities of States on the Moon and Other Celestial Bodies. 1979. URL: http:// disarmament.un.org/treaties/t/moon/text (data obrashcheniya: 01.12.2017).

39. SpaceResources.lu. URL: http://www.spaceresources.public.lu/en/index.html (data obrashcheniya: 01.12.2017).

40. Messier D. Luxembourg Launches Space Resources Mining Initiative // Parabolic Arc. February 03. 2016. URL: http://www.parabolicarc.com/2016/02/03/luxembourg-launches-space-resources-mining-initiative/\#more57416 (data obrashcheniya: 01.12.2017).

41. Messier D. Deep Space Industries Praises Luxembourg Space Resources Mining Initiative // Parabolic Arc. February 03. 2016. URL: http://www.parabolicarc.com/2016/02/03/deep-space-industries-praises-luxembourg-space-resources-mining-initiative/\#more-57417 (data obrashcheniya: 01.12.2017).

42. Messier D. Luxembourg's Bold Move into Space Mining // Parabolic Arc. February 3. 2016. URL: http://www. parabolicarc.com/2016/02/03/luxembourgs-bold-move-space-mining/\#more-57418 (data obrashcheniya: 01.12.2017).

43. Messier D. Luxembourg Commits $\$ 227$ Million to Space Mining Initiative // Parabolic Arc. June 08, 2016. URL: http://www.parabolicarc.com/2016/06/08/luxembourg-commits-227-million-space-mining-initiative/ \#more-58598 (data obrashcheniya: 01.12.2017).

44. Le Luxembourg promulgue la première loi européenne sur l'exploitation des ressources spatiales // Le Monde. 31 Juillet 2017. URL:http://www.lemonde.fr/economie/article/2017/07/31/le-luxembourgpromulgue-la-premiere-loi-europeenne-sur-l-exploitation-des-ressources-spatiales_5167065_3234. html\#T6pwWxU4OF4eG6hJ.99 (data obrashcheniya: 01.12.2017)

45. Hobe S. Was Luxemburg macht, ist krass völkerrechtswidrig // Deutschlandfunk Kultur. 01 Aug. 2017. URL: http://www.deutschlandfunkkultur.de/gesetz-zu-schuerfrechten-im-all-was-luxemburg-macht-ist.1008. de.html?dram:article_id=392506 (data obrashcheniya: 01.12.2017).

46. Luxembourg space exploration laws criticized // Luxembourg Times. 02 Aug. 2017. URL: https://luxtimes. lu/archives/1432-luxembourg-space-exploration-laws-criticised (data obrashcheniya: 01.12.2017).

47. Bel'kova L., Korchmarek N. «Komprometiruet printsip mezhdunarodnogo prava»: «Roskosmos» — o zakone Lyuksemburga o dobyche resursov v kosmose chastnikami // Russia Today. 05 oktyabrya 2017. URL: https:// russian.rt.com/science/article/436642-roskosmos-iskopaemye-kosmos-lyuksemburg (data obrashcheniya: 01.12.2017).

48. Schummer L., Calmes B. Luxembourg law on the exploration and use of space resources entered into force // ARENDT. 02 Aug. 2017. URL: http://www.arendt.com/publications/pages/luxembourg-law-exploration-usespace-resources-into-force.aspx (data obrashcheniya: 01.12.2017).

49. Feider M., Levine T., Graas J. Luxembourg Space Resources Act: Paving the legal road to space // Allen\&Overy. 28 Sept. 2017. URL: http://www.allenovery.com/publications/en-gb/Pages/Luxembourg-Space-ResourcesAct-Paving-the-legal-road-to-space.aspx (data obrashcheniya: 01.12.2017).

50. Thailly L., Schneider F. Luxembourg set to become Europe's commercial space exploration hub with new Space Law. 01 Aug. 2017. URL: http://www.ogier.com/news/the-luxembourg-space-law\# (data obrashcheniya: 01.12.2017).

51. «Izvestiya»: Rossiya predlagaet zapretit' prisvoenie poleznykh iskopaemykh v kosmose // TASS. 11 dekabrya 2017. URL: http://tass.ru/kosmos/4801166 (data obrashcheniya: 01.12.2017).

52. Luxembourg to launch framework to support thr future use of space resources / Released by the Luxembourg Ministry of the Economy. 03 Feb. 2016. URL: http://www.spaceresources.public.lu/content/dam/spaceresources/press-release/2016/2016_02_03PressReleaseAnnouncementSpaceResourceslu.pdf (data obrashcheniya: 01.12.2017).

53. Le gouvernement du Luxembourg et Deep Space Industries ont signé un protocole d'accord pour l'exploration et l'utilisation de ressources spatiales. Communiqué - Publié le 05.05.2016. URL: http://www.gouvernement. lu/5963943/05-ressources-spatiales (data obrashcheniya: 01.12.2017).

54. SpaceResources.lu: Luxembourg Government and Planetary Resources sign MoU to develop activities related to space resource utilization. 13 May 2016. URL: http://www.spaceresources.public.lu/content/dam/ 


\section{Правовое регулирование космической деятельности}

spaceresources/press-release/2016/2016_05_13PressReleaseMoUPR-LuxGvt.pdf (data obrashcheniya: 01.12.2017).

55. Luxembourg and ispace, a Tokyo-based lunar robotic exploration company, sign MoU to co-operate within the SpaceResources.lu initiative. 2 March 2017. URL: http://www.spaceresources.public.lu/content/dam/ spaceresources/press-release/2017/2017_03_02Pressrelease-MoU-iSpace-Lux-Gvt.pdf (data obrashcheniya: 01.12.2017).

56. OHB Venture Capital and LuxSpace establishing company to enable sustainable life in Space. 4 Apr. 2017. URL: http://www.spaceresources.public.lu/en/actualites/2017/ohb-venture-capital-luxspace.html (data obrashcheniya: 01.12.2017).

57. Luxembourg and Kleos Space sign a MoU to co-operate within the SpaceResources.Lu initiative // SpaceResources.Lu. 24 Jul. 2017. URL: http://www.spaceresources.public.lu/en/actualites/2017/News_Kleos_ Space.html (data obrashcheniya: 01.12.2017).

58. Étienne Schneider to Plead in Favour of Multilateral Agreement on Exploration and Use of Space Resources. 21 Apr. 2017. URL: http://www.spaceresources.public.lu/content/dam/spaceresources/press-release/2017/201704-21-press-release-visit-japan.pdf (data obrashcheniya: 01.12.2017).

59. Luxembourg and the European Space Agency enhance cooperation on asteroid missions, related technology and space resources exploration and utilization. 20 June 2017. URL: http://www.spaceresources.public.lu/ content/dam/spaceresources/press-release/2017/2017_06_21\%20Press\%20Release\%20ESA\%20LeBourget. pdf (data obrashcheniya: 01.12.2017).

60. Luxembourg and the United Arab Emirates sign MoU on Space Resources. 10 Oct. 2017. URL: http://www. spaceresources.public.lu/en/actualites/2017/MoU-UAE.html (data obrashcheniya: 01.12.2017).

61. Luxembourg and Japan agree to cooperate on exploration and commercial utilization of Space Resources. 29 Nov. 2017. URL: http://www.spaceresources.public.lu/en/actualites/2017/Luxembourg-and-Japan-Agreeto-Cooperate-on-Exploration-and-Commercial-Utilization-of-Space-Resources.html (data obrashcheniya: 01.12.2017).

62. Luxembourg Government and GomSpace partner to develop new space activities in the Grand Duchy. 17 Sept. 2017. URL: http://www.spaceresources.public.lu/content/dam/spaceresources/press-release/2017/201709-27-press-release-mou-gomspace.pdf (data obrashcheniya: 01.12.2017).

63. Luxembourg government and Spire Global signed cooperation agreement to open an European HQ in the Grand Duchy. 15 Nov. 2017. URL: http://www.spaceresources.public.lu/en/actualites/2017/LuxembourgGovernment-and-Spire-Global-signed-cooperation-agreement-to-open-a-European-HQ-in-the-Grand-Duchy. html (data obrashcheniya: 01.12.2017).

64. SpaceResources.lu: First-time FNR investment in space exploration projects. 23 Nov. 2017. URL: http://www. spaceresources.public.lu/content/dam/spaceresources/press-release/2017/2017-11-23-fnr-space-funding. pdf (data obrashcheniya: 01.12.2017).

65. Masson-Zwaan T. The relevance of hard law and soft law in the further development of space law. 2016. 8 Sept. URL: http://www.unoosa.org/pdf/SLW2016/Panel6/1._Masson-UN_soft_law_hard_law.pdf (data obrashcheniya: 01.12.2017).

66. Schrieberg D. Luxembourg's Bet On Space Industry Shows Early Signs Of Success // The Forbes. 19 Nov. 2017. URL: https://www.forbes.com/sites/davidschrieberg1/2017/11/19/luxembourgs-bet-on-space-industryshows-early-signs-of-success/\#3e9984ec6114 (data obrashcheniya: 01.12.2017).

67. The space industry is now $2 \%$ of Luxembourg's GDP, deputy prime minister Etienne Schneider says // CNBS. 11 Nov. 2017. URL: https://www.cnbc.com/2017/11/11/etienne-schneider-the-space-industry-is-now-2-percent-of-luxembourgs-gdp.html (data obrashcheniya: 01.12.2017). 